\section{ORIGINAL RESEARCH}

D.H. Lee

A. Arat

H. Morsi

H. Shaltoni

J.R. Harris

M.E. Mawad

\title{
Dual Antiplatelet Therapy Monitoring for Neurointerventional Procedures Using a Point-of-Care Platelet Function Test: A Single-Center Experience
}

BACKGROUND AND PURPOSE: Growing evidence of the relationship between poor antiplatelet response and occurrence of clinical events elicited the need of monitoring the response which has not been part of our daily practice. We present our initial experience with a new point-of-care antiplateletfunction test (VerifyNow assay) in neurointerventional procedures.

MATERIALS AND METHODS: Among the 106 consecutive patients from July 2006 to April 2007, ninety-eight met the inclusion criteria. Our preferred antiplatelet regimen was aspirin ( $325 \mathrm{mg}$ daily) and clopidogrel (300 $\mathrm{mg}$ of loading dose followed by $75 \mathrm{mg}$ daily) starting $5-10$ days before the procedure. The test results were reported as aspirin-reaction unit (ARU) for aspirin and $P 2 Y_{12}$ reaction units (PRU), baseline (BASE), and percentage inhibition for the $\mathrm{P}_{2} \mathrm{Y}_{12}$ assay and were summarized as mean \pm SD of the values. We analyzed the effects of several factors of poor clopidogrel response ( $<40 \%$ inhibition). The occurrence of thrombotic events was recorded.

RESULTS: The mean ARU of aspirin assays was $438.3 \pm 47.9$ (range, 350-632), and the response was poor in 2 patients $(2.1 \%)$. For clopidogrel, the mean of the BASE, PRU, and percentage inhibition was $356.8 \pm 56.3$ (range, 234-495), $198.9 \pm 104.4$ (range, 8-401), and $45.2 \pm 27.1 \%$ (range, 0-98), respectively. Forty-two patients (42.9\%) showed poor response. Multivariate analysis showed greater body weight $(81.9 \mathrm{Kg} \pm 19.1 \mathrm{~kg}$ versus $69.9 \pm 15 \mathrm{~kg})$ in the poor-response group. All 3 cases of intraprocedural thrombosis (3.1\%) were observed only in the poor-response group.

CONCLUSION: We observed a high frequency of poor clopidogrel responses in the neurointerventional setting. Routine monitoring of the drug response would be helpful for the early identification of poor antiplatelet responders so that we may modify the regimen and/or treatment plan.

$\mathbf{P}$ latelet inhibition is a prerequisite for the prevention of acute or subacute thrombosis in various neurointerventional procedures such as stent placement in atherosclerotic lesions or stent-assisted coiling of aneurysms. ${ }^{1-5}$ Dual antiplatelet therapy, consisting of aspirin and a thienopyridine, has been used for more than a decade because aspirin monotherapy turned out to be insufficient. ${ }^{6,7}$ The combination of aspirin (75-325 mg daily) and clopidogrel (75 mg daily following a loading dose of $300 \mathrm{mg}$ ) has become the widely accepted standard regimen for stent-placement procedures. ${ }^{8}$

Patients who receive dual antiplatelet therapy plus systemic heparinization, however, may occasionally experience instent thrombosis or a thromboembolic phenomenon. ${ }^{9}$ This may be due to individual variability in the response to antiplatelet treatment. ${ }^{10,11}$ Growing evidence of the relationship between poor response to antiplatelet therapy and the occurrence of clinical events, such as the recurrence of ischemic events and stent-related thrombosis or thromboembolic complications during neurointerventional procedures, elicited the

\section{Received December 7, 2007; accepted after revision January 30, 2008}

From the Department of Radiology and Research Institute of Radiology (D.H.L.), University of Ulsan College of Medicine, Asan Medical Center, Seoul, Korea; Department of Radiology (D.H.L., A.A., H.M., H.S., M.E.M.), Baylor College of Medicine, Houston, Tex; and Accumetrics Inc (J.R.H.), San Diego, Calif.

Abstract previously presented at: 63rd Scientific Assembly and Annual Delegate Meeting of the Korean Radiological Society, Seoul, Korea; October 22-24, 2007

Please address correspondence to Deok Hee Lee, MD, 388-1 Pungnap-dong, Songpa-gu, Seoul 138-736, Korea; e-mail: dhlee@amc.seoul.kr

indicates article with a supplemental on-line table.

DOI 10.3174/ajnr.A1070

need for monitoring the response to antiplatelet agents. ${ }^{12,13}$ Monitoring platelet function, however, has not been part of our daily practice, due to the difficulty and impracticality of laboratory testing. ${ }^{14,15}$

A new point-of-care platelet-function assay (VerifyNow; Accumetrics, San Diego, Calif) enabled us to perform assays to determine the effect of antiplatelet agents. ${ }^{15}$ This study analyzed our initial experiences with this test in the management of patients undergoing various neurointerventional stentplacement procedures.

\section{Materials and Methods}

\section{Patients}

We reviewed our neurovascular prospectively collected data base for consecutive patients pretreated with aspirin and/or clopidogrel for planned or expected stent-placement procedures by the nature of the patient's vascular lesion. Exclusion criteria were the following: 1) unclear antiplatelet medication history, 2) platelet count lower than $150 \times 10^{3} / \mu \mathrm{L}$, and/or 3) hematocrit level lower than $33 \%$ at the time of admission. For a period of 10 months since the availability of the test in our neurovascular angiographic suite (July 2006-April 2007), 106 patients were identified, of whom 98 met inclusion criteria.

There were $72(73.5 \%)$ women and $26(26.5 \%)$ men. Their mean age was $59.1 \pm 15.8$ years. The indications for dual antiplatelet therapy are summarized in Table 1 . Thirty-five patients (35.7\%) did not receive stents due to switching from a stent-assisted to a balloonassisted neck-remodeling method $(n=23)$, no need for neck remodeling $(n=3)$, internal carotid artery trapping $(n=3)$, or deferred treatment $(n=6)$. All stent-placement procedures were performed 


\section{Table 1: Indications for dual antiplatelet therapy}

\section{Indications}

No. $(\%)$

Stent-assisted coiling of aneurysm

$60(61.2)$

Stenting for intracranial artery stenosis

$19(19.4)$

Stenting for extracranial cervical artery stenosis

Stenting for cervical artery dissection

$14(14.3)$

3 (3.1)

Stent-assisted embolization of carotid cavernous fistula

$2(2.0)$

Total

\begin{tabular}{lccc}
\hline \multicolumn{4}{l}{ Table 2: Variability of dual antiplatelet therapy regime } \\
\hline \multicolumn{1}{c}{ Ruration } & Regimen & Days & No. (\%) \\
\hline$>10$ days & ASA + PVX after LD & $<10$ & $31(31.6)$ \\
$5-10$ days & ASA + PVX after LD & $5-10$ & $49(50.0)$ \\
$2-4$ days & ASA + PVX after LD & $2-4$ & $5(5.1)$ \\
1 day & ASA + LD + LD in the morning & 1 & $8(8.2)$ \\
Same day & ASA + 2LDs & 0 & $5(5.1)$ \\
Total & & & $98(100)$ \\
\hline
\end{tabular}

Note:-ASA indicates aspirin $325 \mathrm{mg}$ daily: PVX, clopidogrel $75 \mathrm{mg}$ daily; LD, clopidogrel $300 \mathrm{mg}$ as a loading dose.

with the patient under systemic heparinization regardless of the lesion characteristics. The target-activated clotting time was twice that of the baseline.

\section{Antiplatelet Therapy and Antiplatelet Function Test}

Our preferred antiplatelet regimen was daily aspirin $(325 \mathrm{mg})$ and clopidogrel (75 mg daily following $300 \mathrm{mg}$ of loading dose, Plavix; Sanofi-Aventis, Bridgewater, NJ), starting 5-10 days before the elective stent-placement procedure. The regimen varied, however, according to clinical circumstances. Some patients were already on dual antiplatelet therapy, which was not changed. Other patients could not be pretreated for 5-10 days due to the acuteness of their disease. Patients pretreated for $\leq 2$ days were given an additional $300 \mathrm{mg}$ of clopidogrel on the morning of their procedures, whereas patients admitted for same-day procedures were given $600 \mathrm{mg}$ of clopidogrel plus $325 \mathrm{mg}$ of aspirin immediately before the procedure. Three patients were not given aspirin due to their past history of aspirin-related peptic ulcer disease. Treatment regimens are summarized in Table 2.

Whole blood was obtained either by venipuncture into 1.8mL-draw plastic Vacuette tubes (Greiner, Monroe, NC) containing $3.2 \%$ sodium citrate with a 21 -gauge needle or by transfer to these tubes following a 5-mL discard after obtaining blood from an indwelling catheter. The first tube drawn by venipuncture was labeled as a discard and was at no time used for any experimental assay for platelet function. All tubes containing any anticoagulant other than sodium citrate (eg, ethylenediaminetetraacetic acid for platelet counts) were drawn subsequent to the tubes containing sodium citrate to avoid cross-contamination of samples by an anticoagulant. The tubes were gently inverted $4-5$ times and incubated at room temperature for 10 minutes for the $\mathrm{P} 2 \mathrm{Y}_{12}$ assay and 30 minutes for the aspirin assay.

The VerifyNow assay is a whole-blood point-of-care system that consists of an instrument and single-use assay devices that contain fibrinogen-coated polystyrene beads and a platelet agonist. The basis for the assay is that the beads will agglutinate in whole blood in direct proportion to the number of uninhibited platelets. The assay device is inserted into the instrument, and the whole-blood tube gently inverted $4-5$ times and placed on the device. The instrument measures

the platelet-induced aggregation as an increase in light transmittance and uses a proprietary algorithm to report assay results.

The P2 $\mathrm{Y}_{12}$ device consists of 2 whole-blood assay channels that determine the percentage inhibition of the adenosine diphosphate (ADP)-mediated $\mathrm{P}_{2} \mathrm{Y}_{12}$ receptor. One channel contains fibrinogencoated polystyrene beads and the platelet agonist $\mathrm{ADP}$. ADP is present at a concentration that is equivalent to that observed with $5-\mu \mathrm{mol} / \mathrm{L}$ ADP in studies performed on platelet-rich plasma in an optical aggregometer. Prostaglandin E1 is added to this channel to reduce the nonspecific contribution of the $\mathrm{P}_{2} \mathrm{Y}_{12}$ receptor. The second channel contains fibrinogen-coated polystyrene beads and the platelet-agonist isothrombin-receptor-activating peptide (iso-TRAP). Agglutination in the ADP-containing channel is inhibited by the clopidogrel metabolite, whereas the drug does not inhibit agglutination in the TRAPcontaining channel. The inhibition of the $\mathrm{P}_{2} \mathrm{Y}_{12}$ receptor is calculated by dividing the $\mathrm{P}_{2} \mathrm{Y}_{12}$ reaction unit (PRU) value of the ADP channel by the PRU value of the TRAP channel (a baseline value [BASE]) and converting this value to a percentage (percentage inhibition = [BASE-PRU] / BASE $\times 100[\%])$.

The aspirin device incorporates the platelet agonist arachidonic acid. Aspirin affects hemostasis by the inhibition of platelet function through acetylation of the cyclooxygenase (COX) enzymes. This renders COX-1 incapable of converting arachidonic acid to prostaglandin $\mathrm{H} 2$, a precursor of thromboxane A2 required for normal platelet aggregation. This inhibitory effect provides the basis of the aspirin assay used to measure the pharmacologic effect of aspirin on platelets. Results are reported in aspirin response units (ARU). Individuals not on aspirin without the presence of interfering substances will have ARU values $>550$.

In the absence of an evidence-based cutoff value for the ideal platelet inhibition during the stent-placement procedure, we arbitrarily adopted $<550$ ARU for aspirin and $\geq 40 \%$ inhibition for clopidogrel as indicators of acceptable antiplatelet effect of both drugs. ${ }^{15-19}$ Patients with values below these cutoffs were usually given an additional $300 \mathrm{mg}$ of clopidogrel and/or $325 \mathrm{mg}$ of aspirin via an orogastric tube. Repeat testing was carried out in some patients 30 minutes after the administration of an additional dose.

\section{Analysis and Statistical Tests}

The results of aspirin and clopidogrel assays are reported as mean \pm SD of ARU, BASE, PRU, and percentage inhibition. For clopidogrel response, the patients were divided into 2 groups, those with poor response ( $<40 \%$ inhibition) and those with good response ( $\geq 40 \%$ inhibition), and the effects of age, sex, body weight, presence of risk factors for atherosclerosis, and antiplatelet regimens on clopidogrel response were evaluated. For the statistical analysis, Student $t$ tests were used for continuous variables and $\chi^{2}$ or Fisher exact tests, for categoric variables. To compare the effects of antiplatelet regimens on PRU, we performed multiple comparisons with a Bonferroni correction. Multivariate logistic regression analysis was performed for variables with $P$ values $<.5$. The response to an additional loading dose, which was given in some patients who showed $<40 \%$ of inhibition, was analyzed. For the statistical analyses, the Statistical Package for the Social Sciences K/12.0 (SPSS, Chicago, Ill) was used. A $P$ value $<.05$ was considered statistically significant for all tests except the multiple comparison, for which $<.005$ was used. The occurrence of in-stent thrombosis and/or distal thromboembolism was also noted. 


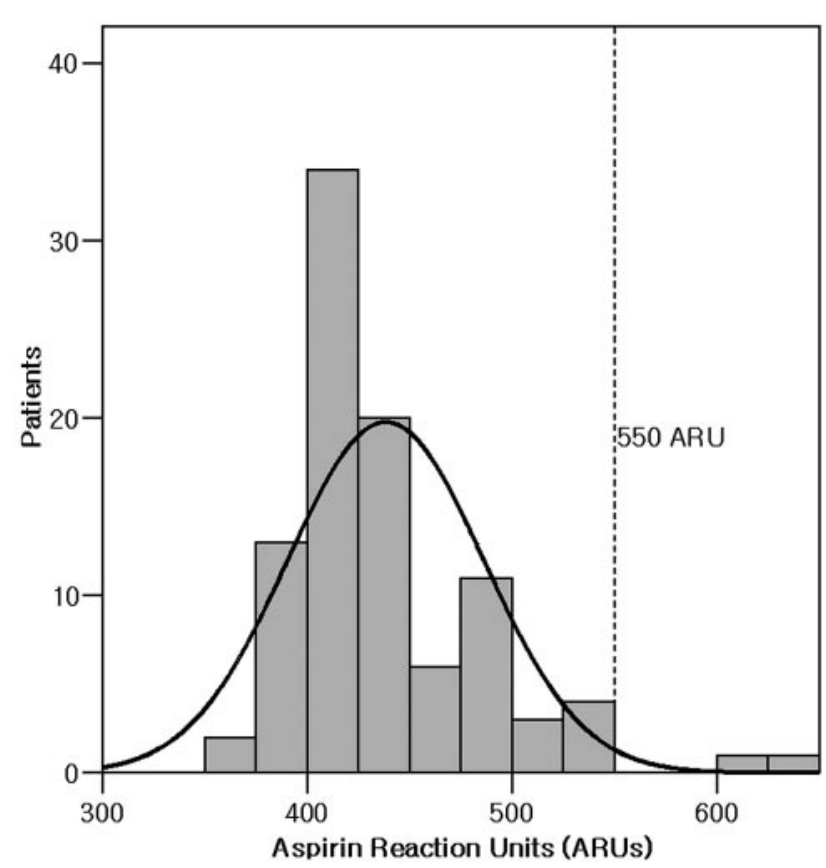

Fig 1. Distribution of ARU in patients receiving dual antiplatelet therapy. Most patients are within the target inhibition range, $<550$ ARU (dotted line)

\section{Results}

\section{Aspirin Response}

Test results were available in 95 patients, who had a mean ARU of $438.3 \pm 47.9$ (range, 350-632 ARU) (Fig 1). Most patients $(98.0 \%)$ except 2 were in the target range for aspirin response, which was $<550$ ARU.

\section{Clopidogrel Response}

The mean of the BASE was $356.8 \pm 56.3$ (range, 234-495) (Fig $2 A$ ), the mean of the PRU was $198.9 \pm 104.4$ (range, $8-401$ ) (Fig $2 B$ ), and the mean of the percentage inhibition was $45.2 \pm$ $27.1 \%$ (range, $0-98$ ) (Fig 2C). With a $<40 \%$ inhibition as a cutoff value, 42 patients $(42.9 \%)$ were in the poor-response group, with the remainder in the good-response group. The mean PRUs of the 5 antiplatelet regimens are shown in Fig 3. The range of PRU values in patients with $<2$ days of antiplatelet treatment varied, and there was a trend toward greater PRU values in patients with $>2$ days but $<5$ days of antiplatelettreatment duration than with other regimens. However, those differences were statistically insignificant.

The results of uni- and multivariate analyses are summarized in On-line Table 1. Univariate analyses showed that the response to the antiplatelet regimen was significantly associated with peripheral platelet count $(P=.002)$ and inversely associated with a patient's weight $(P=.001)$. Multivariate analysis showed that the patient's weight was the only independent factor affecting the response $(P=.01)$.

\section{Repeat Test}

Of the 42 patients in the clopidogrel poor-response group, 29 received an additional loading dose $(300 \mathrm{mg}$ ) of clopidogrel via an orogastric tube; the test was repeated in 10 patients 30 minutes later. Their mean PRU, BASE, and percentage inhibition were $260.3 \pm 112.3,377.3 \pm 75.0$, and $29.4 \pm 31.0 \%$, respectively. Although these PRU and percentage inhibition values showed some improvement relative to the initial values in these patients, these differences were not significant $(P=$ $.064,1.0$, and .160, respectively) (Fig 4).

\section{Intraprocedural Thrombosis and Thromboembolic Events} Although 3 of the 98 patients (3.1\%) experienced intraprocedural thromboses, no symptomatic thromboembolic event was associated with the local thrombosis or the procedure. One patient had an unruptured anterior communicating artery aneurysm and was scheduled to be treated with stentassisted coil embolization. After 10 days of dual antiplatelet therapy, this patient's test results showed 417 ARU, 330 PRU, and $0 \%$ inhibition. To avoid in-stent thrombosis, we embolized this patient's aneurysm by using a balloon-assisted technique, but acute platelet aggregation was observed at the coilparent artery interface. The second patient received a Wingspan stent (Boston Scientific, Fremont, Calif) for symptomatic stenosis of the basilar trunk. After 3 days of dual antiplatelet therapy, this patient had test results of 421 ARU, 318 PRU, and $8 \%$ inhibition; after an additional loading dose $(300$ $\mathrm{mg}$ ) of clopidogrel, this patient had test results showing 378 PRU and $0 \%$ inhibition. We observed acute thrombosis nearly immediately after stent placement. The third patient had an unruptured paraclinoid aneurysm and was treated with stentassisted (Neuroform, Boston Scientific) embolization. After 6 days of antiplatelet therapy, this patient's test results showed 497 ARU, 319 PRU, and 20\% inhibition; after an additional loading dose of clopidogrel, test results showed 235 PRU and $7 \%$ inhibition. A control angiogram obtained 15 minutes after stent placement showed acute in-stent thrombosis. The local thrombosis events in all 3 patients were successfully managed with weight-based intravenous use of abciximab without any clinical consequences.

\section{Discussion}

Applying tentative therapeutic targets of $<550$ ARU for aspirin and $\geq 40 \%$ inhibition for clopidogrel, we found that the incidence of poor response to clopidogrel was quite high $(42.9 \%)$, whereas just a few patients had a poor response to aspirin (2\%).

Clopidogrel, a thienopyridine, is a prodrug, which is converted to active thiol metabolites by hepatic cytochrome $\mathrm{P} 450$ after intestinal absorption. These thiol metabolites irreversibly bind to $\mathrm{P}_{2} \mathrm{Y}_{12}$ receptors on the platelet surface, blocking ADPinduced platelet activation and aggregation. The antiplatelet action of clopidogrel was observed in healthy volunteers as early as 2 hours after a 75-mg dose, with a steady state reached after 5-7 days of daily dosing. A loading dose of $300 \mathrm{mg}$ can shorten the interval of the therapeutic range of the antiplatelet effects of clopidogrel. ${ }^{20}$ A daily dose of $75 \mathrm{mg}$ following a 300 - mg loading dose together with a daily aspirin dose of $80-$ $325 \mathrm{mg}$ has become the standard dosage for stent-placement procedures following the a series of coronary stent-placement trials, which showed that dual antiplatelet therapy was superior to aspirin alone in patients with acute coronary syndrome and after stent implantation. ${ }^{21-23}$

The widespread use of clopidogrel in coronary and cerebral vascular diseases has revealed several negative effects of this drug. Due to its irreversibility in platelet inhibition, patients 

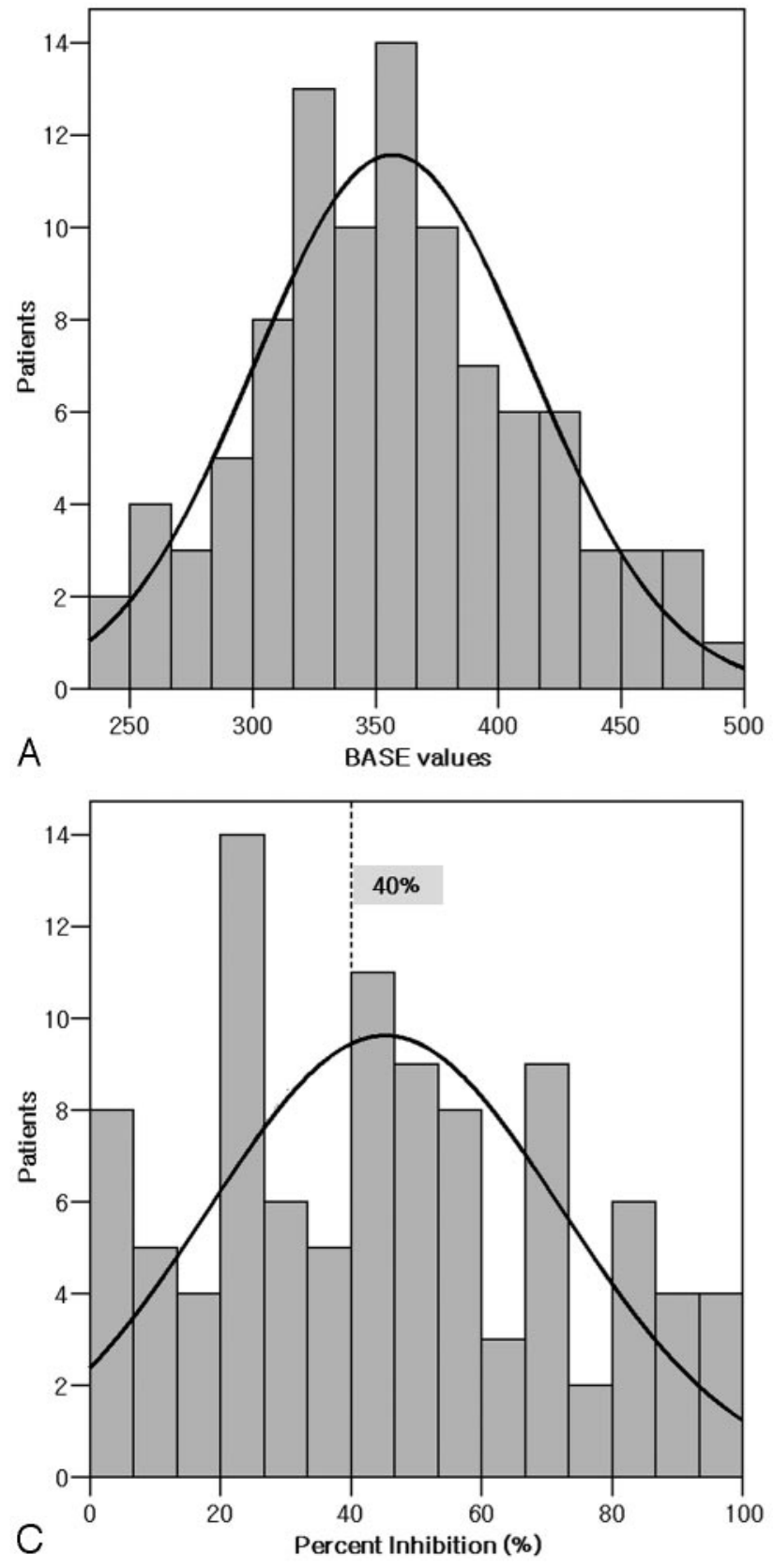

treated with clopidogrel are at higher risk for perioperative bleeding complications, and previous use of clopidogrel interfered with swift conversion to bypass surgery. In addition, though slow onset of antiplatelet action could be overcome by the routine use of a loading dose, there is still a variability in its antiplatelet response. ${ }^{24}$

Clopidogrel resistance, or the occurrence of events despite adequate dosage and patient compliance, has been observed in $4 \%-44 \%$ of patients with coronary artery disease. This variability is due in part to differences in patients tested and in part to differences in the definition of clopidogrel resistance. ${ }^{20}$

Possible causes of clopidogrel resistance could be the following: 1) extrinsic factors, including patient noncompliance, underdosing of the drug, drug-drug interactions, and variability in intestinal absorption or hepatic metabolism; 2) patient conditions that increase platelet reactivity, including diabetes, acute coronary syndrome, and acute stroke; or 3 ) intrinsic

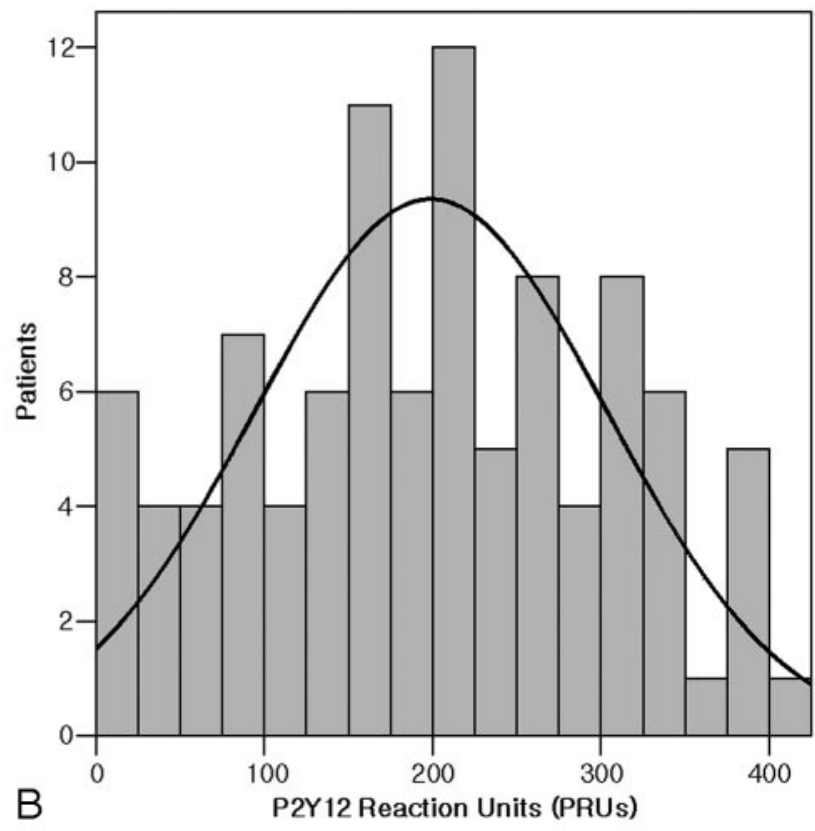

Fig 2. Distribution of values associated with clopidogrel response in patients receiving dual antiplatelet therapy. $A$, BASE value. $B$, PRU. $C$, Percentage inhibition. The working cutoff value is $40 \%$ (dotted line).

factors, including variabilities in $\mathrm{P}_{2} \mathrm{Y}_{12}$ receptor affinity, number of platelet surface receptors, or response to binding. Poor bioavailability of the drug due to reduced intestinal absorption has been suggested as the primary factor. ${ }^{20,25}$

The poor drug effect of clopidogrel could be treated by administering a higher dose to increase its concentration in the blood. For example, a loading dose of $600 \mathrm{mg}$ resulted in significantly lower frequency of resistance, narrower response profile, and rapid antiplatelet effect. However, a loading dose of $900 \mathrm{mg}$ did not show further benefits, due to the ceiling effect of intestinal absorption or metabolism. Although a daily dose of $150 \mathrm{mg}$ may be another solution, ${ }^{26}$ it may be associated with a higher risk of bleeding complications. ${ }^{24} \mathrm{~A}$ new antiplatelet agent (prasugrel), which is said to have more reliable antiplatelet action, is now in clinical trial. ${ }^{27}$

Currently, we are aware of the variability of the antiplatelet action of clopidogrel manifested by occurrence of in-stent thrombosis in the neurointerventional arena. Before the introduction of this point-of-care platelet-function test machine, however, we were not able to verify the phenomenon before the procedure because of limited availability and practicality of standard test methods. The point-of-care and standard tests were shown to correlate, ${ }^{13,14}$ suggesting that the former may be useful in predicting thrombotic events during or after stentplacement procedures. ${ }^{15}$ The routine monitoring of antiplatelet medications has been discussed, with the importance of monitoring, particularly in patients with critical conditions, recently emphasized. ${ }^{28}$ We believe that patients who are involved in stent-placement procedures of the cerebral arteries are candidates for the monitoring of antiplatelet agents.

Most interesting, we observed a relationship between poor response to clopidogrel and patient body weight. To our knowledge, weight-adjusted dosing of clopidogrel has not been recommended. Use of increased loading/maintenance 


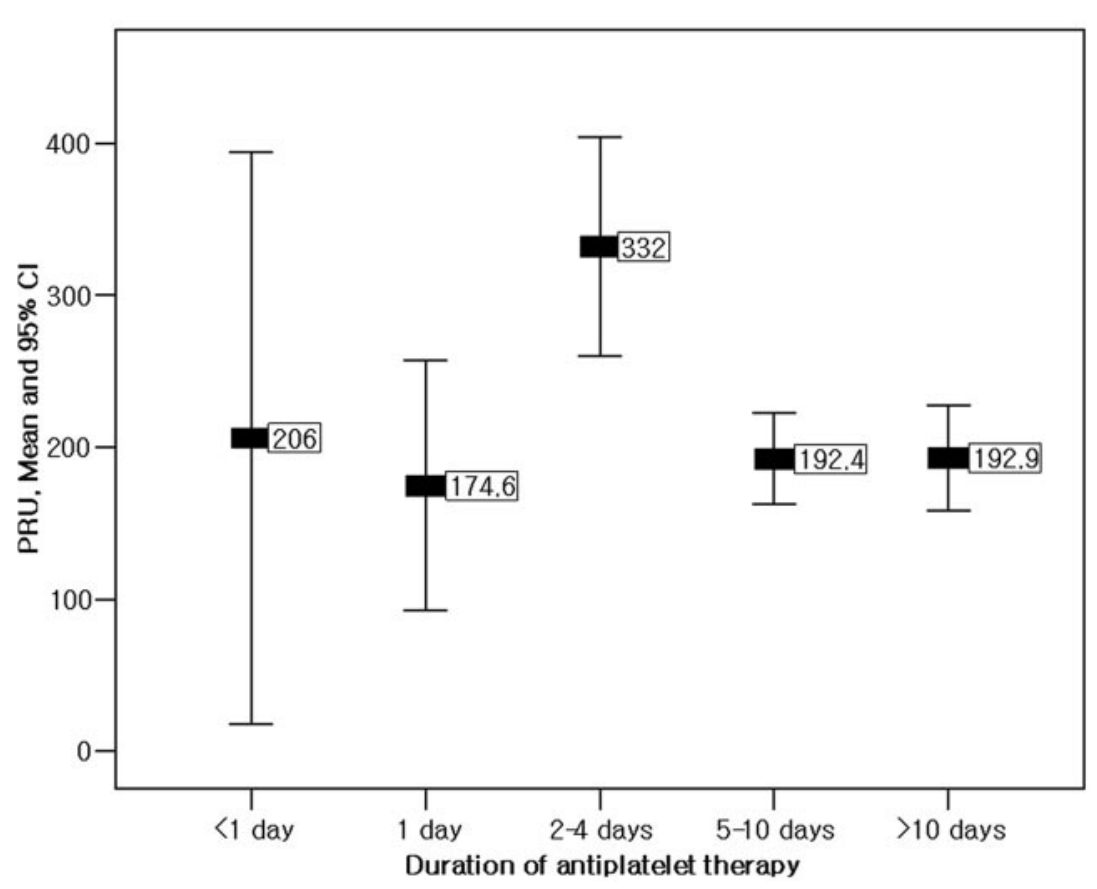

Fig 3. Variability of PRU according to the duration of dua antiplatelet therapy. $\mathrm{Cl}$ indicates confidence interval.
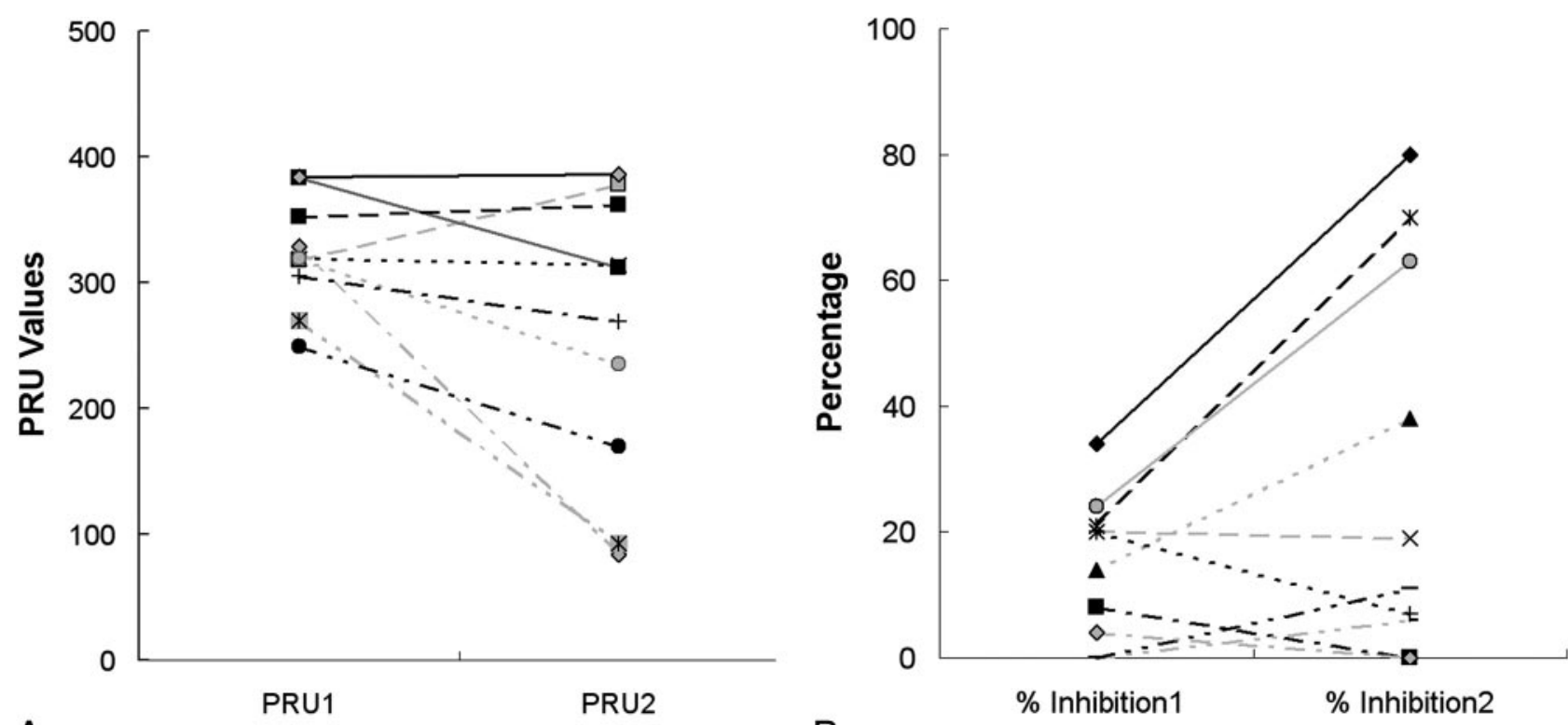

A

B

Fig 4. Changes in PRUs $(A)$ and percentage inhibition (\% inhibition) (B) after an additional clopidogrel loading dose in 10 patients. Results are shown for initial (PRU1 and \% inhibition 1 ) and follow-up tests after an additional loading dose of clopidogrel (PRU2 and \% inhibition2).

doses in patients with increased body weight, however, may provide sufficient inhibition of platelet activity. ${ }^{29}$

Duration of dual antiplatelet therapy can affect antiplatelet activity. ${ }^{24,30}$ We found that long-term administration resulted in a relatively stable level of inhibition. In addition, relatively good inhibition was observed in patients receiving very shortterm administration, though there was significant variability of the response to twofold or additional loading doses. Patients treated for 2-4 days of administration showed the poorest antiplatelet response. A similar trend was observed in a study that performed multiple time point assessments after loading and maintenance doses of clopidogrel. ${ }^{11}$ In elective situations, more than 5 days of premedication would be help- ful for the stable antiplatelet activity of clopidogrel to prevent thrombotic complication with the stent-placement procedure. In nonelective situations, having no less than a 2-hour interval between the administration of clopidogrel and the stent-placement procedure is recommended, considering the pharmacokinetics. It is said that the full antiplatelet effect of the loading dose of clopidogrel occurs after 2 hours of administration. $^{31}$

To avoid the possible risk of acute in-stent thrombosis or thromboembolic complications, we modified our patient management plan according to test results. Patients with $<40 \%$ inhibition were given additional loading doses of clopidogrel before the stent implantation or stents were not im- 
planted. Although our routine interventional procedure included obtaining a control angiogram 20-30 minutes after the completion of the stent-placement procedure, we paid special attention to patients at risk for thrombosis. Procedure-related thromboses occurred in 3 patients, all of whom showed $\leq 20 \%$ inhibition with/without an additional loading dose. Those incidents, however, were managed by the prompt administration of parenteral abciximab, without any clinical consequences.

Our study had several limitations, including its retrospective design and the heterogeneity of the patient group, which included patients with intracranial aneurysms and those with atherosclerotic lesions. In addition, these patients received various dosages of dual antiplatelet treatment for various times. There was no validation of each patient's compliance with the individual antiplatelet regimen, and there was no management algorithm according to test results. However, we believe that this preliminary assessment of our limited experience with this test can help further validate its effectiveness and value in our daily practice. This test could be used not only to prevent thrombotic complications but also to avoid the unnecessary overinhibition of platelet activity that can occur in patients given high-dose regimens.

In conclusion, by using the currently available point-ofcare platelet-function test, we found that the frequency of poor clopidogrel response was high in patients given various dual antiplatelet regimens in the neurointerventional setting. Routine use of point-of-care monitoring, especially in patients scheduled to be treated with stents, can identify poor responders to clopidogrel.

\section{References}

1. Suh DC, Kim SJ, Lee DH, et al. Outcome of endovascular treatment in symptomatic intracranial vascular stenosis. Korean J Radiol 2005;6:1-7

2. Fiorella D, Albuquerque FC, Woo H, et al. Neuroform in-stent stenosis: incidence, natural history, and treatment strategies. Neurosurgery 2006;59:34-42

3. SSYLVIA Study Investigators. Stenting of Symptomatic Atherosclerotic Lesions in the Vertebral or Intracranial Arteries (SSYLVIA): study results. Stroke 2004;35:1388-92. Epub 2004 Apr 22.

4. Ries T, Buhk JH, Kucinski T, et al. Intravenous administration of acetylsalicylic acid during endovascular treatment of cerebral aneurysms reduces the rate of thromboembolic events. Stroke 2006;37:1816-21

5. Grunwald IQ, Papanagiotou P, Politi M, et al. Endovascular treatment of unruptured intracranial aneurysms: occurrence of thromboembolic events. Neurosurgery 2006;58:612-18

6. Bertrand ME, Legrand V, Boland J, et al. Randomized multicenter comparison of conventional anticoagulation versus antiplatelet therapy in unplanned and elective coronary stenting: the full anticoagulation versus aspirin and ticlopidine (fantastic) study. Circulation 1998;98:1597-603

7. Cadroy Y, Bossavy JP, Thalamas C, et al. Early potent antithrombotic effect with combined aspirin and a loading dose of clopidogrel on experimental arterial thrombogenesis in humans. Circulation 2000;101:2823-28

8. Yan BP, Clark DJ, Ajani AE. Oral antiplatelet therapy and percutaneous coronary intervention. Expert Opin Pharmacother 2005;6:3-12

9. Cutlip DE. Stent thrombosis: historical perspectives and current trends. $J$ Thromb Thrombolysis 2000;10:89-101
10. Gurbel PA, Tantry US. Aspirin and clopidogrel resistance: consideration and management. J Interv Cardiol 2006;19:439-48

11. Serebruany VL, Steinhubl SR, Berger PB, et al. Variability in platelet responsiveness to clopidogrel among 544 individuals. J Am Coll Cardiol 2005;45: 246-51

12. Siller-Matula J, Schror K, Wojta J, et al. Thienopyridines in cardiovascular disease: focus on clopidogrel resistance. Thromb Haemost 2007;97:385-93

13. von Beckerath N, Pogatsa-Murray G, Wieczorek A, et al. Correlation of a new point-of-care test with conventional optical aggregometry for the assessment of clopidogrel responsiveness. Thromb Haemost 2006;95:910-11

14. van Werkum JW, van der Stelt CA, Seesing TH, et al. A head-to-head comparison between the VerifyNow P2Y12 assay and light transmittance aggregometry for monitoring the individual platelet response to clopidogrel in patients undergoing elective percutaneous coronary intervention. J Thromb Haemost 2006;4:2516-18. Epub 2006 Aug 28

15. Malinin A, Pokov A, Spergling M, et al. Monitoring platelet inhibition after clopidogrel with the VerifyNow-P2Y12(R) rapid analyzer: the VERIfy Thrombosis risk ASsessment (VERITAS) study. Thromb Res 2007;119:277-84. Epub 2006 Mar 24

16. Coleman JL, Wang JC, Simon DI. Determination of individual response to aspirin therapy using the accumetrics Ultegra RPFA-ASA system. Point of Care 2004;3:77-82

17. Malinin A, Spergling M, Muhlestein B, et al. Assessing aspirin responsiveness in subjects with multiple risk factors for vascular disease with a rapid platelet function analyzer. Blood Coagul Fibrinolysis 2004;15:295-301

18. Angiolillo DJ, Fernandez-Ortiz A, Bernardo E, et al. Identification of low responders to a 300 -mg clopidogrel loading dose in patients undergoing coronary stenting. Thromb Res 2005;115:101-08

19. Patrono C, Coller B, FitzGerald GA, et al. Platelet-active drugs: the relationships among dose, effectiveness, and side effects - the Seventh ACCP Conference on Antithrombotic and Thrombolytic Therapy. Chest 2004;126(suppl 3):234S-64S

20. Gurbel PA, Tantry US. Clopidogrel resistance? Thromb Res 2006;120:311-21

21. Mehta SR, Yusuf S, Peters RJ, et al. Effects of pretreatment with clopidogrel and aspirin followed by long-term therapy in patients undergoing percutaneous coronary intervention: the PCI-CURE study. Lancet 2001;358:527-33

22. Steinhubl SR, Berger PB, Mann JT, et al. Early and sustained dual oral antiplatelet therapy following percutaneous coronary intervention: a randomized controlled trial. JAMA 2002;288:2411-20

23. Yusuf S, Zhao F, Mehta SR, et al. Effects of clopidogrel in addition to aspirin in patients with acute coronary syndromes without ST-segment elevation. N Engl J Med 2001;345:494-502

24. Gurbel PA, Bliden KP, Hayes KM, et al. The relation of dosing to clopidogrel responsiveness and the incidence of high post-treatment platelet aggregation in patients undergoing coronary stenting. J Am Coll Cardiol 2005;45:1392-96

25. Wiviott SD. Clopidogrel response variability, resistance, or both? Am J Cardiol 2006;98:18N-24N

26. von Beckerath N, Kastrati A, Wieczorek A, et al. A double-blind, randomized study on platelet aggregation in patients treated with a daily dose of 150 or 75 mg of clopidogrel for 30 days. Eur Heart J 2007;28:1814-19

27. Wiviott SD, Braunwald $\mathrm{E}$, McCabe $\mathrm{CH}$, et al. Prasugrel versus clopidogrel in patients with acute coronary syndromes. N Engl J Med 2007;357:2001-15. Epub 2007 Nov 4

28. Smith SC Jr, Feldman TE, Hirshfeld JW Jr, et al. ACC/AHA/SCAI 2005 guideline update for percutaneous coronary intervention: a report of the American College of Cardiology/American Heart Association Task Force on Practice Guidelines (ACC/AHA/SCAI Writing Committee to Update 2001 Guidelines for Percutaneous Coronary Intervention). Circulation 2006;113:e166-286

29. Feher G, Koltai K, Alkonyi B, et al. Clopidogrel resistance: role of body mass and concomitant medications. Int J Cardiol 2006;120:188-92

30. Gurbel PA, Bliden KP, Hiatt BL, et al. Clopidogrel for coronary stenting: response variability, drug resistance, and the effect of pretreatment platelet reactivity. Circulation 2003;107:2908-13

31. Hochholzer W, Trenk D, Frundi D, et al. Time dependence of platelet inhibition after a 600-mg loading dose of clopidogrel in a large, unselected cohort of candidates for percutaneous coronary intervention. Circulation 2005;111: $2560-64$ 